\title{
THE POLYNOMIAL IDENTITIES OF THE GRASSMANN ALGEBRA
}

\author{
BY
}

\section{KRAKOWSKI AND A. REGEV}

ABSTRACT. By using the theory of codimensions the $T$-ideal of polynomial identities of the Grassmann (exterior) algebra is computed.

1. Introduction. An important question in P.I. algebras is to describe the $T$-ideal of identities of a given P.I. algebra. The problem seems to be quite difficult and up to this point the only known cases are the identities for the commutative and nilpotent algebras. In this paper the question is answered for the case of the Grassmann algebra using a method which seems open to generalization.

2. The codimensions of the Grassmann algebra. The Grassmann (exterior) algebra $E$ over any field $F$ of characteristic $\not 2$ is generated by the sequence of elements $\left\{1, e_{1}, e_{2}, \cdots\right\}$ together with the identity $e_{i} e_{j}+e_{j} e_{i}=0$. Hence $D=$ $\{1\} \cup\left\{e_{i_{1}} \cdots e_{i_{m}} \mid 1 \leq i_{1}<i_{2}<\cdots<i_{m}\right\}$ is a basis for $E$. We define the length of a basis element $1 \neq a=e_{i_{1}} \cdots e_{i_{m}} \in D$ to be $m$. The following facts are immediate:

(1) If $a \in D$ has even length then $a \in Z(E)$, the center of $E$.

(2) If $a, b \in D$ both have odd length, then $a b=-b a$.

Using (1) and (2), one proves

(3) $[[x, y], z]=0$ for any $x, y, z \in E$, where $[[]$,$] is the Jacobi polynomial,$ $[x, y]=x y-y x$.

For any choice of $n$ elements in $D,\left\{a_{1}, \cdots, a_{n}\right\}$, let $I \subseteq\{1, \cdots, n\}$ denote those indices for which $a_{i}$ is of odd length. Let $\sigma \in S_{n}$, the symmetric group on $n$ elements. Then by (1) and (2) we have that $a_{\sigma_{1}} \cdots a_{\sigma_{n}}=\left(f_{I}(\sigma)\right) a_{1} \cdots a_{n}$, where $f_{I}(\sigma) \in\{ \pm 1\}$.

The function $f$ is therefore computed as follows: since the permutations of an ordered set are in one-to-one correspondence with the reorderings of the set, $\sigma \in S_{n}$ determines a reordering of the $n$-tuple $(1,2, \cdots, n)$ from its natural order to $\left(\sigma_{1}, \cdots, \sigma_{n}\right)$. Now consider an arbitrary subset $I \subseteq\{1, \cdots, n\}$ and $\sigma \in S_{n}$. The reordering $\sigma$ on $\{1, \cdots, n\}$ induces a reordering of the subset $I$ and hence, by the one-to-one correspondence, yields a permutation on $I$. (Note that this does

Received by the editors September 21, 1972.

AMS (MOS) subject classifications (1970). Primary 16A38. 
not imply that $\sigma(I) \subseteq I$.) It can easily be shown that the sign of this induced permutation is $f_{I}(\sigma)$. For example, if $n=\{1,2, \cdots, 5\}, \sigma=\left(\begin{array}{lllll}1 & 2 & 3 & 4 & 5 \\ 2 & 4 & 5 & 3 & 1\end{array}\right)$ and $I=$ $\{1,3,5\}$, then the subset $I$ has been reordered by $\sigma$ from the ordered triple $(1,3,5)$ to $(5,3,1)$. This corresponds to the permutation $\left(\begin{array}{lll}1 & 3 & 5 \\ 5 & 3 & 1\end{array}\right)$ on $I$ and hence $f_{l}(\sigma)=-1$.

From the above we have $f: 2^{n} \times S_{n} \rightarrow\{ \pm 1\}$. We shall be concerned with the $2^{n} \times n$ ! matrix $H=\left(f_{I}(\sigma)\right)$ which shall be determined by ordering the $2^{n}$ subsets of $\{1, \ldots, n\}$ and using these as row indices, and ordering the $n$ ! permutations of $S_{n}$ and using these as column indices. When dealing specifically with the $m$ th case we shall write $H$ as $H^{(m)}=\left(f_{I}^{(m)}(\sigma)\right) ; I \subseteq\{1, \ldots, m\}$ and $\sigma \in S_{m}$.

As in (1) we shall define $V_{n}(x)=\operatorname{Sp}_{F}\left\{x_{\sigma_{1}} \cdots x_{\sigma_{n}} \mid \sigma \in S_{n}\right\}$ where $x_{i} \in\{x\}$, an infinite set of noncommutative indeterminates. $F[x]$ shall denote the free ring in $\{x\}$ over $F$. The codimension $d_{n}$ of the $T$-ideal $Q \subseteq F[x]$ is

$$
d_{n}=\operatorname{dim}_{F} \frac{V_{n}(x)}{Q \cap V_{n}(x)} .
$$

Lemma (2.1). Let $\left\{d_{n}\right\}$ be the codimension sequence for the T-ideal $Q$ of the polynomial identities of the Grassmann algebra. Then $d_{n}=\operatorname{rank}_{F} H^{(n)}$.

Proof. Let $g\left(x_{1}, \cdots, x_{n}\right) \in V_{n}(x)$. By multilinearity, $g\left(x_{1}, \cdots, x_{n}\right)$ is in $Q$ if and only if $g$ vanishes on all the basis elements in $D$. Write $g\left(x_{1}, \cdots, x_{n}\right)=$ $\Sigma_{\sigma \in S_{n}} \alpha_{\sigma} x_{\sigma_{1}} \cdots x_{\sigma_{n}}$ and consider the $\alpha_{\sigma}$ as unknowns. Let $\left\{a_{i}\right\}_{i=1}^{n} \subseteq D$ and substitute

$$
\begin{aligned}
g\left(a_{1}, \ldots, a_{n}\right) & =\sum_{\sigma \in S_{n}} a_{\sigma} a_{\sigma} \cdots a_{\sigma}=\sum_{\sigma \in S_{n}} a_{\sigma}\left(f_{I}^{(n)}(\sigma)\right) a_{1} \cdots a_{n} \\
& =\left(\sum_{\sigma \in S_{n}} a_{\sigma} f_{l}^{(n)}(\alpha)\right) a_{1} \cdots a_{n} .
\end{aligned}
$$

So $g\left(x_{1}, \cdots, x_{n}\right)$ is an identity if and only if $\Sigma_{\sigma \in S_{n}} a_{\sigma} f_{I}^{(n)}(\sigma)=0$ for every $I \subseteq\{1, \cdots, n\}$. This yields a set of $2^{n}$ linear equations in $n !$ unknowns $\left\{\bar{a}_{\sigma}\right\}_{\sigma \in S_{n}}$, with the $2^{n} \times n !$ matrix of coefficients $=H^{(n)}=\left(f_{l}^{(n)}(\sigma)\right) ; \sigma \in S_{n}$, $I \subseteq\{1, \ldots, n\}$. The dimension of the solution space of the above linear equations is $n !-\operatorname{rank}_{F} H^{(n)}$ and this is also the dimension of $Q \cap V_{n}(x)$. Now $\operatorname{dim} V_{n}(x)=$ $n !$, hence $d_{n}=n !-\operatorname{dim}_{F}\left(Q \cap V_{n}(x)\right)=n !-\left(n !-\operatorname{rank}_{F} H^{(n)}\right)^{n}=\operatorname{rank}_{F} H^{(n)}$. Q.E.D.

We define some notations to be used in this section:

$$
\left(i_{1}, \cdots, i_{n}\right)=\left(\begin{array}{llll}
1 & 2 & \cdots & n \\
i_{1} & i_{2} & \cdots & i_{n}
\end{array}\right) \in S_{n} .
$$

For $\sigma=\left(i_{1}, \cdots, i_{n}\right) \in S_{n},(\sigma, n+1)$ shall indicate the permutation $\left(i_{1}, \cdots, i_{n}\right.$, $n+1) \in S_{n+1}$; similarly for $\sigma \in S_{n-1}$ we define $(\sigma, n, n+1) \in S_{n+1}$. If $\sigma=$ $\left(i_{1}, \cdots, i_{j-1}, n, i_{j+1}, \cdots, i_{n}\right) \in S_{n}$, then we indicate the replacement of $n$ by 
$n+1$ as $\sigma(n \hat{,} n+1)=\left(i_{1}, \cdots, i_{j-1}, n+1, i_{j+1}, \cdots, i_{n}\right)$ (this is not, as it stands, a permutation). Thus, if $\sigma \in S_{n}$, then $(\sigma(n \hat{,}, n+1), n) \in S_{n+1}$ exchanges the positions of $n$ and $n+1$ in $(\sigma, n+1)$.

Instead of working with $H^{(n)}$ we define, by induction, a sequence of $2^{n-1} \times$ $2^{n-1}$ submatrices $M^{(n)}$ of $H^{(n)}$ by choosing and ordering a subset of $2^{n-1}$ permutations of $S_{n}$ and $2^{n-1}$ subsets of $\{1, \cdots, n\}$. This is done as follows: Begin with

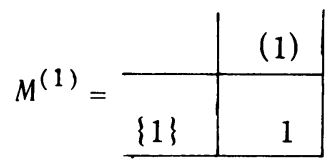

and assume that $M^{(n)}$ has been defined with columns indexed by $\sigma^{(j)} \in S_{n}, j=$ $1,2, \cdots, 2^{n-1}$, and rows indexed by $I_{j} \subseteq\{1, \cdots, n\}, j=1,2, \cdots, 2^{n-1}$. The $2^{n}$ permutations indexing the columns of $M^{(n+1)}$ are

$$
\gamma(k)= \begin{cases}\left(\sigma^{(k)}, n+1\right) & \text { for } 1 \leq k \leq 2^{n-1}, \\ \left(\sigma^{\left(k-2^{n-1}\right)}\left(n^{n}, n+1\right), n\right) & \text { for } 2^{n-1}<k \leq 2^{n} .\end{cases}
$$

For the $2^{n}$ row indices we have

$$
J_{k}= \begin{cases}I_{k} & \text { for } 1 \leq k \leq 2^{n-1}, \\ I_{k-2^{n-1}} \cup\{n+1\} & \text { for } 2^{n-1}<k \leq 2^{n}\end{cases}
$$

(it follows easily by induction that $J_{k}$ is a row index if and only if $1 \in J_{k}$ ). Thus, for example, we obtain $M^{(3)}$ from $M^{(2)}$ :

$V^{(2)}=$\begin{tabular}{c|c|c|} 
& $(1,2)$ & $(2,1)$ \\
\hline$\{1\}$ & 1 & 1 \\
\hline$\{1,2\}$ & 1 & -1 \\
\hline
\end{tabular}

$\rightarrow M^{(3)}=$\begin{tabular}{c|c|c|c|c|} 
& $(1,2,3)$ & $(2,1,3)$ & $(1,3,2)$ & $(3,1,2)$ \\
\hline$\{1\}$ & 1 & 1 & 1 & 1 \\
\hline$\{1,2\}$ & 1 & -1 & 1 & 1 \\
\hline$\{1,3\}$ & 1 & 1 & 1 & -1 \\
\hline$\{1,2,3\}$ & 1 & -1 & -1 & 1 \\
\hline
\end{tabular}

Lemma (2.2). For $1 \in I \subseteq\{1, \cdots, n\}$ and $\sigma \in S_{n^{\prime}}$

(a) $f_{I}^{(n)}(\sigma)=f_{I}^{(n+1)}(\sigma, n+1)=f_{l \cup\{n+1\}}^{(n+1)}(\sigma, n+1)$.

For $1 \in I \subseteq\{1, \cdots, n-1\}$ and $\sigma \in S_{n-1}$

(b) $f_{I}^{(n-1)}(\sigma)=f_{I}^{(n+1)}(\sigma, n+1, n)=f_{l \cup\{n+1\}}^{(n+1)}(\sigma, n+1, n)$,

(c) $f_{I}^{(n-1)}(\sigma)=f_{l \cup\{n\}}^{(n+1)}(\sigma, n+1, n)=-f_{l \cup\{n, n+1\}}^{(n+1)}(\sigma, n+1, n)$. 
For $1 \in I \subseteq\{1, \cdots, n-1\}$ and $\sigma \in S_{n}$

(d) $f_{I}^{(n)}(\sigma)=f_{I}^{(n+1)}(\sigma(n, n+1), n)$,

(e) $f_{I \cup\{n\}}^{(n)}(\sigma)=f_{I \cup\{n\}}^{(n+1)}(\sigma(n \hat{,}, n+1, n)$.

Proof. The method of computing $f_{I}(\sigma)$ is as follows: Let $\sigma=\left(i_{1}, \ldots, i_{n}\right) \in S_{n}$ and $l \subseteq\{1, \cdots, n\}$. Remove from the ordered set $\left(i_{1}, \ldots, i_{n}\right)$ those integers in the complementary set $\{1, \cdots, n\}-I$ and consider the remaining letters as determining a reordering of their natural order. This reordering determines a permutation whose sign is then $f_{I}(\sigma)$. Claims (a)-(e) now become immediate. For example, to prove that $f_{I}^{(n)}(\sigma)=f_{I}^{(n+1)}(\sigma, n+1)$ we write $\sigma=\left(i_{1}, \cdots, i_{n}\right)$ and note that removing $\{1, \cdots, n\}-I$ from $\left\{i_{1}, \cdots, i_{n}\right\}$ leaves the same result as removing $\{1, \cdots, n, n+1\}-I$ from $\left\{i_{1}, \cdots, i_{n}, n+1\right\}$; hence the two signs are equal. Similar arguments yield the other statements of the lemma.

Lemma (2.3). The rank of $H^{(n)} \geq 2^{n-1}$.

Proof. It is sufficient to show that the rows of $M^{(n)}$ are linearly independent for all $n$. The case $n=1$ (and $n=2$ ) is true by inspection. Assume the result is true for $M^{(r)}, 1 \leq r \leq n$.

We describe $M^{(n+1)}$ by beginning with $M^{(n-1)}$ and using two inductive steps: Let $I_{1}, \cdots, I_{2 n-2}$ be the row indices for $M^{(n-1)}$. Then $I_{1}, \cdots, I_{2 n-2}, I_{1} \cup$ $\{n\}, \cdots, I_{2 n-2} \cup\{n\}$ are the row indices for $M^{(n)}$, and hence the row indices for $M^{(n+1)}$ are

$$
\begin{gathered}
I_{1}, \ldots, I_{2}{ }_{2-2}, I_{1} \cup\{n\}, \ldots, I_{2^{n-2}} \cup\{n\}, I_{1} \cup\{n+1\}, \cdots, \\
I_{2}{ }^{n-2} \cup\{n+1\}, I_{1} \cup\{n, n+1\}, \cdots, I_{2}{ }^{n-2} \cup\{n, n+1\} .
\end{gathered}
$$

Similarly, let $\sigma^{(1)}, \ldots, \sigma^{\left(2^{n-2}\right)}$ be the column indices for $M^{(n-1)}$. Then those for $M^{(n)}$ are

$$
\begin{gathered}
\left(\sigma^{(1)}, n\right), \ldots,\left(\sigma^{\left(2^{n-2}\right)}, n\right) \\
\left(\sigma^{(1)}(n-1 \hat{1}, n), n-1\right), \ldots,\left(\sigma^{\left(2^{n-2}\right)}\left(n-1^{\wedge}, n\right), n-1\right)
\end{gathered}
$$

and for $M^{(n+1)}$ we obtain

$$
\begin{gathered}
\left(\sigma^{(1)}, n, n+1\right), \ldots,\left(\sigma^{\left(2^{n-2}\right)}, n, n+1\right), \\
\left(\sigma^{(1)}(n-1 \hat{1}, n), n-1, n+1\right), \ldots,\left(\sigma^{\left(2^{n-2}\right)}(n-1 \uparrow, n), n-1, n+1\right), \\
\left(\sigma^{(1)}, n+1, n\right), \ldots,\left(\sigma^{\left(2^{n-2}\right)}, n+1, n\right), \\
\left(\sigma^{(1)}(n-1 \wedge, n+1), n-1, n\right), \ldots,\left(\sigma^{\left(2^{n-2}\right)}(n-1 \wedge, n+1), n-1, n\right) .
\end{gathered}
$$

Partitioning the index sets in this manner determines the following division 
of $M^{(n+1)}$ into four quarters, and of two of the quarters into 8 subquarters:

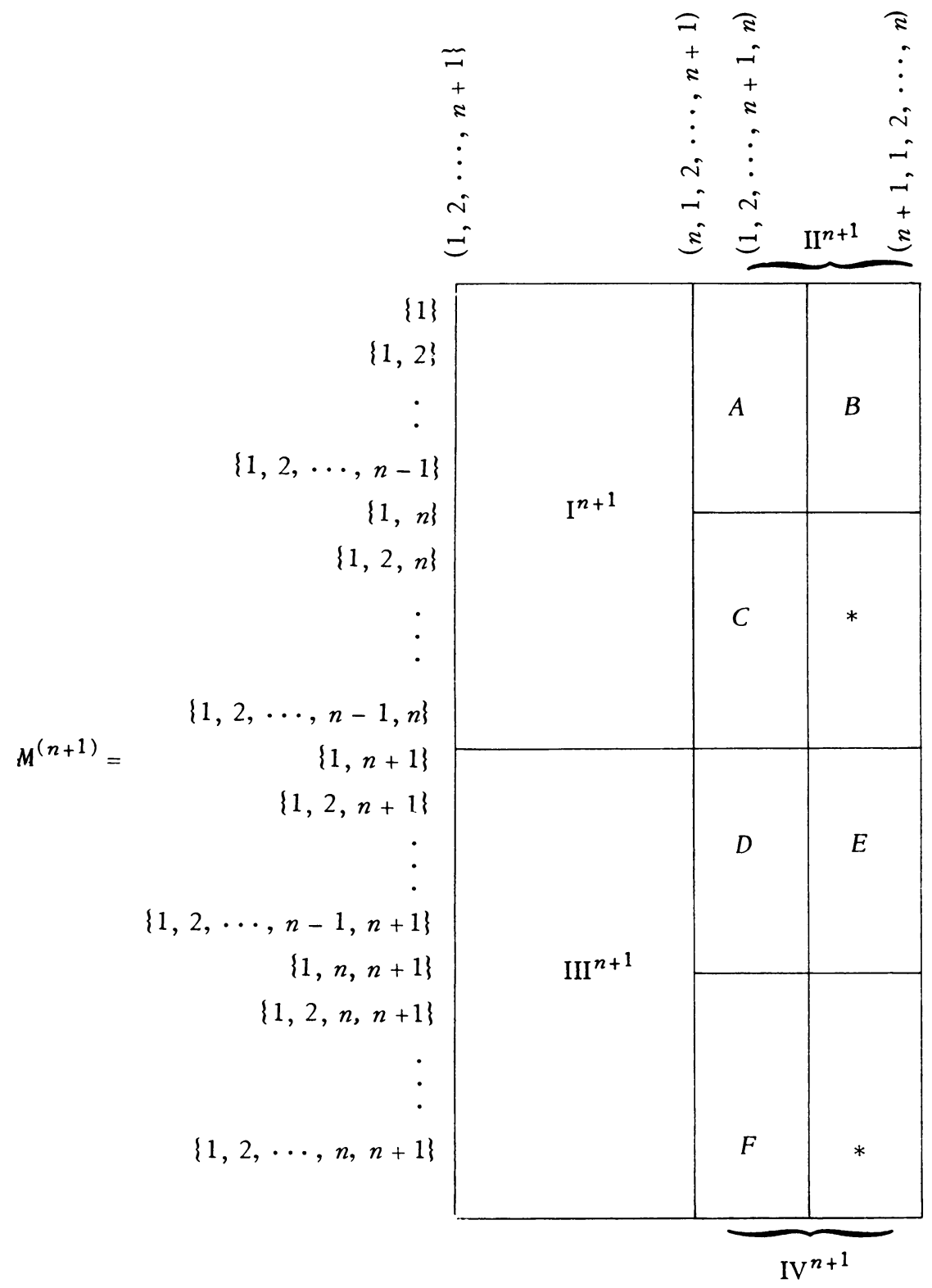

The following facts are a restatement of Lemma (2.2):

Sublemma. (i) $\mathrm{I}^{n+1}=\mathrm{III}^{n+1}=M^{(n)}$.

(ii) $A=D=M^{(n-1)}$.

(iii) $C=-F=M^{(n-1)}$.

(iv) $B=I I^{n}$.

(v) $E=I^{n}$. 
Proof. We prove only (iv); the rest are similar. With the notations above, and by the manner of construction of the $M^{(n)}$ s, $\mathrm{II}^{n}$ is the matrix determined by the row indices $I_{1}, \cdots, I_{2 n-2} \subseteq\{1, \cdots, n-1\}$ and column indices $\left(\sigma^{(1)}(n-\hat{1, n)}\right.$, $n-1), \cdots,\left(\sigma^{\left(2^{n-2}\right)}\left(n^{2 n-1, n) n}-1\right)\right.$. Now $B$ is determined by the same row indices and by the column indices $\left(\sigma^{(1)}(n-1, n+1), n-1, n\right), \cdots$, $\left(\sigma^{\left(2^{n-2}\right)}(n-1, n+1), n-1, n\right)$. So we must show that for $\sigma \in S_{n}$ and $I \subseteq\{1, \cdots, n-1\}$,

$$
f_{I}^{(n)}(\sigma(n-1, \hat{,}), n-1)=f_{I}^{(n+1)}(\sigma(n-\hat{1, n}+1), n-1, n) .
$$

But this is exactly the content of Lemma (2.2d) with $\sigma=(\sigma(n-1, n), n-1)$.

Proof of the Lemma. Since $\mathrm{I}^{n+1}=M^{(n)}$, the upper half of $M^{n+1}$ has linearly independent rows, by induction. Upon rowwise subtraction of the upper half of $M^{(n+1)}$ from the lower half we obtain (using the Sublemma) in the lower half

$$
L=\left(\begin{array}{c|c|c}
0 & \mathrm{IV}^{n}-\mathrm{II}^{n} \\
\hline-2 M_{n-1} & *
\end{array}\right) .
$$

Now

$$
M^{(n)}=\left(\begin{array}{c|c}
\mathrm{I}^{n} & \mathrm{II}^{n} \\
\hline \mathrm{III}^{n} & \mathrm{IV}^{n}
\end{array}\right) .
$$

By similar arguments $\mathrm{I}^{n}=\mathrm{III}^{n}=M^{(n-1)}$. Therefore by rowwise subtraction of the upper half from the lower half we obtain the matrix

$$
\left(\begin{array}{c|c}
\mathrm{I}^{n} & \mathrm{II}^{n} \\
\hline 0 & \mathrm{IV}^{n}-\mathrm{II}^{n}
\end{array}\right) .
$$

By induction, $M^{(n-1)}$ and $M^{(n)}$ have linearly independent rows and hence it follows that $\mathrm{IV}^{n}-\mathrm{II}^{n}=E-B$ has independent rows. Therefore $L$, and hence $M^{(n+1)}$, has independent rows. Q.E.D.

3. Codimensions of $J_{d}$ type algebras. Let $F$ be a field. An algebra satisfying an identity of the form $x_{1} \cdots x_{d}=\Sigma_{\sigma \epsilon S_{d} \sigma_{1 F 1}} \alpha_{\sigma \sigma_{\sigma_{1}}} \cdots x_{\sigma_{d}}$ will be called an algebra of type $J_{d}$.

The basic reference for this section will be [1]. The definitions and notations to be used will be found in the first 15 lines of $\$_{1}$ of [1]; that is, the definitions of $V_{n}(x), V_{n}^{(t)}(x), U_{n}^{(t)}(x)$. As in Definition (1.5), [1], the codimension $d_{n}$ of the $T$-ideal $Q \subseteq F[x]$ is

$$
d_{n}=\operatorname{dim}_{F} \frac{V_{n}(x)}{Q \cap V_{n}(x)} .
$$


Following Lemma (1.7) in [1] with $Q_{n}(x)=Q \cap V_{n}(x)$ replacing $W(l, n)$ we have Lemma (3.1).

$$
d_{n}=\sum_{t=1}^{n} \operatorname{dim}_{F} \frac{V_{n}^{(t)}+U_{n}^{(t)}+Q_{n}}{U_{n}^{(t)}+Q_{n}} .
$$

We shall denote $\operatorname{dim}_{F}\left(V_{n}^{(k)}+U_{n}^{(k)}+Q_{n}\right) /\left(U_{n}^{(k)}+Q_{n}\right)$ as $a_{(n)}^{(k)}$. Let $1 \leq t \leq n$. We shall make use of the short notation $\left(\hat{x}_{t}\right)=\left(x_{1}, \cdots, \hat{x}_{t}, \cdots, x_{n}\right)=\left(x_{1}, \cdots\right.$, $\left.x_{t-1}, x_{t+1}, \cdots, x_{n}\right)$. Let

$$
V_{n-1}\left(\hat{x}_{t}\right)=\operatorname{Sp}\left\{x_{\sigma} \cdots x_{\sigma_{n-1}} \mid\left\{\sigma_{1}, \cdots, \sigma_{n-1}\right\}=\{1, \cdots, t-1, t+1, \cdots, n\}\right\}
$$

denote the space spanned by all the $(n-1)$ ! multilinear monomials in $\left(\hat{x}_{t}\right)$. Let $\phi$ be the isomorphism induced by the correspondence $y_{1} \rightarrow x_{1}, \ldots, y_{t-1} \rightarrow x_{t-1}$, $y_{t} \rightarrow x_{t+1}, \cdots, y_{n-1} \rightarrow x_{n}$. Then $\phi\left(V_{n-1}(y)\right)=V_{n-1}\left(\hat{x}_{t}\right)$ and denote the image $\phi\left(Q_{n-1}(y)\right)$ by $Q_{n-1}\left(\hat{x}_{t}\right)$. It is obvious that $x_{t} V_{n-1}\left(\hat{x}_{t}\right)=V_{n}^{(t)}(x)$, and that $x_{t} Q_{n-1}\left(\hat{x}_{t}\right) \subseteq Q_{n}(x)$.

Theorem (3.1). Let $A$ be an algebra of type $J_{d}$, with $\left\{d_{n}\right\}$ its sequence of codimensions. Then, for any $n, d_{n} \leq(d-1)^{n-1}$.

For the proof we shall need three lemmas and the following preliminaries: Let $n$ be any positive integer, $1 \leq k \leq n$, and let the monomial $M(x)=x_{k} x_{\sigma_{2}} \cdots$ $x_{\sigma_{n}} \in V_{n}^{(k)}(x)$. Denote the $s=n-k+1$ indices which satisfy $\sigma_{k} \geq k$ by $\mu_{1}, \cdots, \mu_{s}$ according to their order of appearance in the monomial $M(x)$. The other indices will be indicated by $x_{i_{j}}$ according to their order of appearance after $\mu_{j}$ so that $\left(k=\mu_{1}\right), M(x)=\left(x_{\mu_{1}} x_{1}{ }_{1} \cdots x_{1_{r_{1}}}\right)\left(x_{\mu_{2}} x_{2_{1}} \cdots x_{2_{r_{2}}}\right) \cdots\left(x_{\mu_{s}} x_{s_{1}} \cdots x_{s_{r_{s}}}\right)$. The mapping

$$
\begin{gathered}
y_{1} \rightarrow\left(x_{\mu_{1}}{ }^{x_{1}} \cdots x_{1}\right) \\
\cdot \\
\cdot \\
y_{s} \rightarrow\left(x_{r_{1}} x_{s}{ }^{s_{1}} \cdots x_{s_{r_{s}}}\right)
\end{gathered}
$$

induces an isomorphism $\psi: V_{s}(y) \rightarrow V_{n}(x)$ which is an into linear transformation. Using this notation we have the following lemma, whose proof is immediate:

Lemma (3.2). (a) $M(x) \in \psi\left(V_{s}^{(1)}(y)\right) \subseteq V_{n}^{(k)}(x)$.

(b) $\psi\left(U_{s}^{(1)}(y)\right) \subseteq U_{n}^{(k)}(x)$.

(c) $\psi\left(Q_{s}(y)\right) \subseteq Q_{n}(x)$.

Lemma (3.3). Let $A$ be an algebra of type $J_{d}$. Let $d \leq n$ and $1 \leq k \leq n-d+1$. Then $a_{(n)}^{(k)}=0$. 
Proof. We show $V_{n}^{(k)} \subseteq U_{n}^{(k)}+Q_{n}$. It is sufficient to prove the result for the monomials spanning $V_{n}^{(k)}$. Let $M(x)=x_{k^{x} \sigma_{2}} \cdots x_{\sigma_{n}} \in V_{n}^{(k)}(x)$, and $\psi$ be as above. $k \leq n-d+1 \Rightarrow d \leq n-k+1=s$. Since $A$ is of type $J_{d}$ it satisfies $y_{1} \cdots y_{d}=\Sigma_{\sigma_{1} \neq 1} a_{\sigma} y_{\sigma_{1}} \cdots y_{\sigma_{d}}$. Since $d \leq s$ we can multiply both sides by $y_{d+1} \cdots y_{s}$ obtaining an identity for $A$ of the form $y_{1} \cdots y_{s}=\Sigma_{\sigma_{1} \neq_{1}} a_{\sigma} y_{\sigma_{1}} \cdots y_{\sigma_{s}}$. Each term and hence the sum of the right-hand side is obviously contained in $U_{s}^{(1)}(y)$, so that $y_{1} \cdots y_{s} \in U_{s}^{(1)}(y)+Q_{s}(y)$. Hence

$$
M(x)=\psi\left(y_{1} \cdots y_{s}\right) \in \psi\left(U_{s}^{(1)}(y)\right)+\psi\left(Q_{s}(y)\right) \subseteq U_{n}^{(k)}(x)+Q_{n}(x) . \quad \text { Q.E.D. }
$$

Lemma (3.4). For $1 \leq k \leq n, a_{(n)}^{(k)} \leq d_{n-1}$.

Proof. By definition, $d_{n-1}=\operatorname{dim}_{F}\left(V_{n-1}(y)+Q_{n-1}(y)\right) / Q_{n-1}(y)$. Applying the isomorphism $\phi$ we obtain $d_{n-1}=\operatorname{dim}_{F}\left(V_{n-1}\left(\hat{x}_{k}\right)+Q_{n-1}\left(\hat{x}_{k}\right)\right) / Q_{n-1}\left(\hat{x}_{k}\right)$. Multiplication on the left by $x_{k}$.induces an isomorphism of $V_{n-1}\left(\hat{x}_{k}\right)$ onto $V_{n}^{(k)}(x)$ sending $Q_{n-1}\left(\hat{x}_{k}\right)$ onto $x_{k} Q_{n-1}\left(\hat{x}_{k}\right)$. Hence

$$
\begin{aligned}
& d_{n-1}=\operatorname{dim}_{F} \frac{x_{k} V_{n-1}\left(\hat{x}_{k}\right)+x_{k} Q_{n-1}\left(\hat{x}_{k}\right)}{x_{k} Q_{n-1}\left(\hat{x}_{k}\right)} \\
& \quad \geq \operatorname{dim}_{F} \frac{V_{n}^{(k)}(x)+Q_{n}(x)}{Q_{n}(x)} \geq \operatorname{dim}_{F} \frac{V_{n}^{(k)}+U_{n}^{(k)}(x)+Q_{n}(x)}{U_{n}^{(k)}(x)+Q_{n}(x)}=a_{(n)}^{(k)} \text {. Q.E.D. }
\end{aligned}
$$

Proof of the Theorem. If $n \leq d-1$ we are finished, for in this case $d_{n} \leq n$ ! $\leq(d-1)^{n-1}$. Assume $n \geq d$ and induct: $d_{n-1} \leq(d-1)^{n-2}$. By the lemmas above

$$
d_{n}=\sum_{k=1}^{n} a_{(n)}^{(k)}=\sum_{k=n-d+2}^{n} a_{(n)}^{(k)} \leq(d-1) d_{n-1} \leq(d-1)^{n-1} \text {. Q.E.D. }
$$

Corollary. Let $\left\{d_{n}\right\}$ be the codimension sequence for the Grassmann algebra E. Then $d_{n}=2^{n-1}$.

Proof. Since $E$ satisfies $[[x, y], z], E$ is a $J_{3}$ type algebra and hence $d_{n} \leq 2^{n-1}$. Using Lemmas (2.1) and (2.3) we conclude that $d_{n} \geq 2^{n-1}$, and hence $d_{n}=2^{n-1}$.

4. Applications. It is well known that the existence of a polynomial identity implies the existence of a homogeneous, multilinear identity [2]. By the multilinear part of a $T$-ideal we shall mean the vector space of all multilinear identities in the ideal. For a polynomial $f \in F[x]$ we shall denote the $T$-ideal generated by $f$ as $T(f)$.

The following lemma is known but its proof is difficult to extract from the literature and so we include it here. 
Lemma (4.1). Let $F$ bave characteristic 0 , and $P, Q \subseteq F[x]$ be two T-ideals whose multilinear parts are the same. Then $P=Q$.

Proof. Let $M(P)$ be the multilinear part of $P$. So $M(P)=M(Q)$. Let $b\left(x_{1}, \cdots, x_{n}\right) \in F[x]$ have degree of $x_{i}=d_{i}$, and $t_{b}=\max \left\{d_{i}\right\}$. We assume $f \in P$ and show $f \in Q$. Let $t=t_{f}$ and consider $\Delta_{t}=\left\{b \in P \mid t_{b} \leq t\right\}$. For any $b \in \Delta_{t}$ define the sequence $r(b)=\left(r_{1}, \cdots, r_{t}\right)$ where $r_{j}=$ the number of variables of degree $j$ in $b$.

The $t$-tuples thus defined are completely ordered by the right lexicographic order. We shall show $f \in Q$ by applying $t$-tuple induction.

Write $f=\Sigma_{\nu} f_{\nu}$ as a sum of homogeneous polynomials in every variable, and since $F$ is an infinite field, each $f_{\nu} \in P$. We shall show that $f_{\nu} \in Q$ and hence we may assume that $f$ itself is homogeneous.

Assume that $x_{i}$ has degree $t$ in $f$. If $t=1 f$ is already multilinear, hence in $Q$. So $t>1$ and we may assume that $x_{1}$ has degree $t$. In the multilinearization process one defines $[2$, p. 224]

$$
\begin{aligned}
& g\left(u, v, x_{2}, \cdots, x_{n}\right) \\
& \quad=f\left(u+v, x_{2}, \cdots, x_{n}\right)-f\left(u, x_{2}, \cdots, x_{n}\right)-f\left(v, x_{2}, \cdots, x_{n}\right) .
\end{aligned}
$$

Hence $g \in T(f)$. But $g\left(u, u, x_{2}, \cdots, x_{n}\right)=2\left(2^{t-1}-1\right) f\left(u, x_{2}, \cdots, x_{n}\right)$ and since char $F \neq 0$ and $t>1,2\left(2^{t-1}-1\right) \neq 0$ and so $f \in T(g)$. Hence $T(f)=T(g)$. If $f \rightarrow r(f)=\left(r_{1}, \cdots, r_{t}\right)$ then $g \rightarrow r(g)=\left(s_{1}, \cdots, s_{t}\right)$ with $s_{t} \leq r_{i}$ Now $g \in P$, $g$ is lexicographically comparable and precedes $f$ in the order, hence by the $k$ tuple induction, $g \in Q$ and so $f \in Q$. Q.E.D.

Let $P$ denote the $T$-ideal of identities of the Grassmann algebra $E$, and $Q=$ $T([[x, y], z])$.

Theorem (4.1). For the Grassmann algebra $E$ over a field $F$ of characteristic $\neq 2$ the codimensions of $P=$ the codimensions of $T([[x, y], z])=Q$, and $P_{n}=Q_{n}$.

Proof. Since $E$ satisfies the identity $[[x, y], z]$ we have that $Q \subseteq P$. Hence

$$
P_{n}(x)=P \cap V_{n}(x) \supseteq Q \cap V_{n}(x)=Q_{n}(x) \Rightarrow \operatorname{dim}_{F}\left(V_{n} / Q_{n}\right) \geq \operatorname{dim}_{F}\left(V_{n} / P_{n}\right),
$$

and these are the codimensions. Hence, by Lemmas (2.1), (2.3) and the corollary to Theorem (3.1),

$$
\begin{aligned}
2^{n-1} & \geq \operatorname{dim}_{F}\left(V_{n} / Q_{n}\right) \geq \operatorname{dim}_{F}\left(V_{n} / P_{n}\right) \geq 2^{n-1}, \\
\operatorname{dim}_{F} P_{n} & =\operatorname{dim}_{F} Q_{n}=n !-2^{n-1} .
\end{aligned}
$$

Now $P_{n} \supseteq Q_{n} \Rightarrow P_{n}=Q_{n}$. Q.E.D.

Corollary. If char $F=0$ then $P=Q$. 
Proof. $Q_{n}$ and $P_{n}$ are just the multilinear homogeneous polynomials in $n$ variables in $P$ and $Q$. Since the se are $T$-ideals $M(P)=M(Q)$ and so by Lemma (4.1), $P=Q$. Q.E.D.

Let $T=\left(\left[\left[\ldots\left[\left[x_{1}, x_{2}\right], x_{3}\right] \ldots\right], x_{n}\right]\right)$ be the $T$-ideal generated by the generalized Jacobi polynomial. Let $F[x] / T$ be the universal algebra for this identity and $\left\{c_{n}\right\}$ be its sequence of codimensions. Then we conjecture that, as in Theorem (3.1), $c_{n}=(d-1) !(d-1)^{n-d+1}$.

\section{REFERENCES}

1. A. Regev, Existence of identities in $A \otimes B$, Israel J. Math. 11 (1972), 131-152.

2. N. Jacobson, Structure of rings, 2nd rev. ed., Amer. Math. Soc. Colloq. Publ., vol. 37, Amer. Math. Soc., Providence, R. I., 1956. MR 18, 373.

THE WEIZMANN INSTITUTE OF SCIENCE. REHOVOT, ISRAEL

Current address (D. Krakowski): Department of Mathematics, Wayne State University, Detroit, Michigan 48202

Current address (A. Regev): Department of Mathematics, University of California, Los Angeles, California 90024 